\title{
Calculation of Gaussian Quadrature with High Accuracy Mathematics Package
}

\section{H. Poghosyan}

NAS RA V. Ambartsumian Byurakan Astrophysical Observatory (BAO), Armenia E-mail:poghosyan.hovhannes@gmail.com

\begin{abstract}
We are checking here the dependence of numerical integration accuracy on the quantity of integration points and the accuracy of machine representation of numbers. For this purpose, the package HAHMath is applied. This package allows one to carry out calculations with arbitrary long machine decimal numbers, presented as vectors of integers. Integrals are substituted for Gaussian sums where Hermite polynomials zeros and corresponding weights, computed by the same package are used. It is shown that the chosen case accuracy of final calculations depends on the used machine numbers' length more strictly than on the quantity of the integration points.
\end{abstract}

Keywords: Numerical calculations - Gaussian quadrature - Hermite polynomials: zeros and weights.

\section{Introduction}

While carrying out numerical computing one can encounter a need for calculation of definite integral taken from function between some limits. Undoubtedly, each case of such calculation requires individual solution, depending on the properties of the integration elements. Nevertheless, one can classify quadrature procedures starting with trapezium rule and Simpsons rule and up to highest order Newton-Cotes formulae, which can serve as appropriate numerical methods for integration of a rather wide class of functions.

On the other hand, the method of Gaussian quadrature is a more, let us say, function-depending procedure. It depends on the integrand properties and therefore the quadrature should be chosen carefully according the type of expression under the integral sign and limits of integration.

In the present paper, we intend to verify the dependence of the accuracy of substitution of the integral by the quadrature sum on the number of summation points (on the order of the polynomial, zeros of which are used) and on the number of significant digits in the machine representation of numbers. Since in the sequel we will be interested in calculating integrals of expressions that are given on an infinite 
range of argument values, while having a specific dependence appropriate for interpolation by the Hermite polynomials, we use Gaussian quadrature defined using the zeros and weights of the mentioned polynomial.

\section{Gaussian quadrature}

The word quadrature means numerical integration. Speaking about a quadrature one means numerical integration of definite integrals. The replacement of a definite integral by a quadrature formula is a common technique in the numerical solution of mathematical problems. There are various quadrature methods and any particular technique must be chosen based on the form of the integrand.

Any Gaussian quadrature has a purpose to obtain the best numerical estimate of an integral by picking optimal abscissas $x_{i}$ for the best evaluating the function $f(x)$. The fundamental theorem of Gaussian quadrature states the optimal abscissas of the $m$-point Gaussian quadrature formulas are precisely the roots of orthogonal polynomial for the same interval and weighting function. Gaussian quadrature is optimal because it fits all polynomials up to degree $2 m-1$ exactly.

For Gauss Quadrature Rule first we need specify an infinite, complete, orthogonal sequence of polynomials where the domain of the polynomials matches the desired integration interval. The rule is states as below

$$
\int_{a}^{b} f(x) d x \approx \sum_{i=1}^{m} f\left(x_{i}\right) w_{i}
$$

One can obtain good result producing such substitution if the function $f(x)$ can be well approximated by a polynomial function within the integration range $(a, b)$. If the integrated function can be written in the form as follows: $f(x)=g(x) w(x)$ where $g(x)$ is polynomial and $w(x)$ is determined then according to Gauss Quadrature Rule integral (1) can be calculated as follows:

$$
\int_{a}^{b} f(x) d x=\int_{a}^{b} g(x) w(x) d x=\sum_{i=1}^{m} f\left(x_{i}\right) w_{i}
$$

where the quantities $x_{i}$, called zeros represent the roots of the chosen polynomial and $w_{i}$ are weights for given quadrature.

The problems, we are going to study involving numerical calculations deal with integrands, which have special forms given in the infinite interval $(-\infty,+\infty)$, and one can use for their computing, so called, Gauss-Hermite quadrature. Moreover, taking 
into account that expressions under the integral are even functions, one can calculate the integrals in the interval $(0,+\infty)$ only. Then we conclude that one should apply the following computational scheme for our purposes:

$$
\int_{0}^{+\infty} f(x) d x=\int_{0}^{+\infty} \exp \left(-x^{2}\right) f(x) d x=\sum_{i=0}^{m} f\left(x_{i}\right) w_{i} .
$$

Therefore, one needs to calculate, the quantities $\left\{x_{i}\right\}$ and $\left\{w_{i}\right\}$ for computing the required integrals given by (3). For this purpose, one should find zeros of Hermite polynomials of various orders. One can find can find lists of the mentioned quantities in the literature, however those lists are only for limited orders of the polynomials (see, for example, Abramowitz \& Stegun 1979).

\section{Hermite polynomials}

Hermite polynomials form an orthogonal system and are among the most extensively studied polynomials. These polynomials arethe solutions of the following linear, second-order ordinary differential equation:

$$
y^{\prime \prime}-2 x y^{\prime}+2 n y=0,
$$

where $n$ is a non-negative integer and $y^{\prime}$ and $y^{\prime \prime}$ are the first and second derivatives $y(x)$ function. It is easy to see that the polynomial of $n$-th order derived using the following expression:

$$
H_{n}(x)=(-1)^{n} \exp \left(x^{2}\right) \frac{d^{n}}{d x^{n}} \exp \left(-x^{2}\right)
$$

for any non-negative $n$ is a solution for equation (4).We list below the first five Hermite polynomials

$$
\begin{gathered}
H_{0}(x)=1, \\
H_{1}(x)=2 x, \\
H_{2}(x)=4 x^{2}-2, \\
H_{3}(x)=8 x^{3}-12 x, \\
H_{4}(x)=16 x^{4}-48 x^{2}+12 .
\end{gathered}
$$


One can immediately verify that all these functions are solutions of the equation (4). These polynomials have some important properties, which makes them very useful for various fundamental and practical implementations. First, as we mentioned it above, these polynomials make up an orthogonal with the weight function $e^{-x^{2}}$ and complete system on the infinite interval $(-\infty,+\infty)$. This property makes them a very useful tool for the expansion into series of some type of functions over these polynomials.

The orthogonality condition for the Hermite polynomials will have the following general form:

$$
\int_{-\infty}^{\infty} \exp \left(-x^{2}\right) H_{n}(x) H_{m}(x) d x=\sqrt{\pi} 2^{m} m !, \text { if } m=n
$$

and

$$
\int_{-\infty}^{\infty} \exp \left(-x^{2}\right) H_{m}(x) H_{n}(x) d x=0 \text {, if } m \neq n .
$$

These integrals showing the orthogonality condition, on the other hand, can be used for testifying the numerical integration accuracy. In one of our next papers devoted to the numerical calculations' accuracy, we are going to consider this issue in its general form. Here we will check it for a particular case only.

\section{Numerical results}

All numerical procedures are carried out applying the package HAHMath compiled on FORTRAN programming language. This package compiled by $\mathrm{H}$. Harutyunian in 1992,allows one producing computations with many significant digits, decreasing thus accumulation of errors inevitable otherwise due to machine rounding of numbers. All numbers in this package are given as vectors consisting of single-byte integers, the sequence of which represents a number. For providing precise results of numerical computing, long type integers are used in this package. In computer science long integer means a data type, which has range greater than the standard data type integer and represented as a set of binary digits.

Each integer in the common vector storages four consecutive digits of a long number. This representation of the numbers puts its special requirements on the organization of computer calculations. In particular, one needs to perform all arithmetic operations using special separate subroutines. It makes computational programs rather complicated. On the other hand, this package creates a flexible computing environment if one needs to perform calculations with numbers of 
several tens or hundreds of significant digits. All subroutines are testified using calculations repeatedly. As a handy test for implementation of multiple computation, the package has been successfully used for calculation of the number $\pi$ with 160 significant digits.

Using the program package HAHMath we calculated with very high accuracy the needed zeros $\left\{x_{i}\right\}$ and corresponding weights $\left\{w_{i}\right\}$ for Hermite polynomials of the required order. For this calculation, the method of progressive approximation was used. It allows one finding the polynomial roots with desired accuracy, provided, that numerical computations guarantee the required accuracy. Since the length of the used numbers is an input parameter, one always can choose it according to the requirements.

After these sets of numbers are ready, one can use them for calculation of integrals. Here we make some simple calculations for checking the dependence of the computation results on both the number of integration points (or polynomial order) and the accuracy of number representation (the quantity of significant digits in the used numbers).

If we put in the equation (6) $m=n=0$ we will obtain

$$
\int_{-\infty}^{\infty} \exp \left(-x^{2}\right) d x=\sqrt{\pi}
$$

Having the numerical value of $\pi$, one can check easily how changes the substitution of integral (8) by its Gaussian quadrature the accuracy of calculations. It is obvious, that there are two factors, which determine the accuracy of the quadrature calculation accuracy. One of those factors, as we mentioned above, the order of the interpolation polynomial. And second one is the numbers' length or the quantity of digits in the representation of the used numbers. No doubts, the integrand in the relation (8) has a simple form, which suggests that in this case no need for extremely high order polynomials. However, one can assert it for sure only having implemented the numerical experiment. Hence, we carried out all the necessary calculations for checking the accuracy dependence on the parameters enumerated above.

Thus, making the following substitution of the integral in the equation (8) by a Gaussian quadrature one will obtain

$$
\int_{0}^{\infty} \exp \left(-x^{2}\right) d x=\frac{\sqrt{\pi}}{2} \approx \sum_{i=1}^{m} w_{i} \exp \left(-x_{i}^{2}\right) .
$$

Carrying out the corresponding calculations, we obtained a set of results for various orders of polynomials and different lengths of the used numbers. In the Table below, 
we show the results of computations in the form of differences between the values of $\pi$ obtained by direct calculations and having all the used digits correct and using the approximate formula given by the expression (9). For this purpose, we used "long numbers" characterized by various significant digits, designed by $L(10,20,40,60,80$ and 100) and zeros of polynomials of order $\mathrm{N}(10,20,30,100)$.

As one can see from the Table, the length of numbers is more essential, at least for such kind calculations, where the integrand has a simple form. The accuracy of calculations practically does not depend on the number of quadrature integration points (the order of polynomials).

Table 1. The accuracy of calculations carried out according the formula (9).

\begin{tabular}{|c|c|c|c|c|}
\hline $\mathbf{N}$ & 10 & 20 & 30 & 100 \\
\hline 10 & $1.18 \mathrm{D}-09$ & $-1.45 \mathrm{D}-10$ & $-3.17 \mathrm{D}-10$ & $-2.77 \mathrm{D}-10$ \\
20 & $-3.26 \mathrm{D}-20$ & $-3.95 \mathrm{D}-20$ & $-2.75 \mathrm{D}-20$ & $-3.60 \mathrm{D}-20$ \\
40 & $4.98 \mathrm{D}-40$ & $2.91 \mathrm{D}-40$ & $2.58 \mathrm{D}-40$ & $6.51 \mathrm{D}-41$ \\
60 & $2.36 \mathrm{D}-60$ & $-1.48 \mathrm{D}-60$ & $2.61 \mathrm{D}-60$ & $-4.08 \mathrm{D}-60$ \\
80 & $-3.16 \mathrm{D}-80$ & $1.44 \mathrm{D}-80$ & $6.71 \mathrm{D}-81$ & $-1.70 \mathrm{D}-80$ \\
100 & $-3.80 \mathrm{D}-101$ & $-5.55 \mathrm{D}-100$ & $-2.31 \mathrm{D}-100$ & $-9.64 \mathrm{D}-100$ \\
\hline
\end{tabular}

\section{Conclusion}

Substitution of definite integrals by Gaussian quadrature gives rather good results for the integrands having a multiplier of the type $e^{-x^{2}}$. It is important to notice that the main impact on calculations have our given accuracy for the used numbers or their length. It is easy to see that all results given in the Table 1 are of the same order in each row independently of the polynomial's order. On the contrary, the results in the columns differ from each other drastically. Therefore, one arrives at a conclusion that in these calculations the number length has a crucial significance.

Acknowledgement. The author thanks H.A.Harutynian for valuable discussions and his help in applying the programming package HAHMath.

\section{Reference}

Abramowitz, M., Stegun, I., 1979, Handbook of Mathematical Functions, Nauka (in Russian) 